\title{
The role of anterior segment optical coherence tomography (OCT) in the diagnosis of corneal hydrops post penetrating keratoplasty in a patient with keratoconus
}

\author{
AL-Damri AL-Jawhara ${ }^{*}$, Alkharashi Majed ${ }^{2}$ \\ ${ }^{1}$ King Saud Medical City, Riyadh, Saudi Arabia \\ ${ }^{2}$ College of Medicine, King Saud University, Riyadh, Saudi Arabia
}

\begin{abstract}
Objectives: (1) To report the case of a patient post penetrating keratoplasty (PKP) for keratoconus and developed corneal hydrops near the junction between the donor cornea and the host cornea; (2) to show the value of optical coherence tomography (OCT) of the anterior segment in diagnosing such cases; and (3) to review the literature about acute corneal hydrops post PKP.

Case: A 52-year-old woman who post PKP in her right eye for keratoconus 32 years ago. She was referred to Anterior segment clinic in King saud university hospital with the diagnosis of endothelial rejection. She had been experiencing blurred vision and photophobia in her right eye for 5 days.

Results: Examination of the right eye showed a visual acuity of 20/400, $12 \mathrm{~mm} \mathrm{Hg}$ intraocular pressure, no ciliary injection, and quiet conjunctiva. She had a large (about $9 \mathrm{~mm}$ ) corneal graft with an inferotemporal area of ectasia and stromal edema near the junction of the donor cornea and the host cornea. There were neither keratic precipitates nor anterior chamber reaction. The lens showed early nuclear sclerosis cataract. Anterior segment OCT showed Descemet membrane detachment. In her left eye, the patient had a visual acuity of 20/200, normal intraocular pressure, and corneal ectasia, with apical scarring. The lens showed early nuclear sclerosis cataract. Examination of the fundus revealed normal findings in both eyes.
\end{abstract}

Conclusions: Acute corneal hydrops post PKP is a rare condition that should be included in the differential diagnosis of graft edema in a quiet eye. Anterior segment OCT is a very helpful tool in diagnosing this condition, eliminating the use of unnecessary steroid therapy.

\section{Introduction}

Corneal hydrops is a known complication of keratoconus, resulting from a break in the Descemet membrane (DM) that allows aqueous to enter the corneal stroma and epithelium. The edema usually resolves in 8-12 weeks with subsequent scarring in cases of acute hydrops. The incidence of corneal hydrops has been reported as being $2.6 \%-3 \%$ in patients with keratoconus, $6 \%-11 \%$ in pellucid marginal corneal degeneration, and $11 \%$ in keratoglobus [1-3]. In this article, we describe a case of corneal hydrops post PKP that was misdiagnosed and treated as endothelial rejection, and value of optical coherence tomography (OCT) of anterior segment structures in diagnosing such conditions. We also review the literature regarding the incidence and management of acute hydrops post PKP in patients with keratoconus.

\section{Case}

This report concerns a 52-year-old woman post PKP in her right eye for keratoconus 32 years ago. She was referred to Anterior segment clinic in King saud university hospital with the diagnosis of endothelial rejection. She had been experiencing blurred vision and photophobia in her right eye for 5 days. Examination of the right eye showed a visual acuity of 20/400, $12 \mathrm{~mm} \mathrm{Hg}$ intraocular pressure, no ciliary injection, and quiet conjunctiva. She had a large (about $9 \mathrm{~mm}$ ) corneal graft with an inferotemporal area of ectasia and stromal edema near the junction of the donor cornea and the host cornea. There were neither keratic precipitates nor anterior chamber reaction.
The lens showed early nuclear sclerosis cataract. In her left eye, the patient had a visual acuity of $20 / 200$, normal intraocular pressure, and corneal ectasia, with apical scarring. The lens showed early nuclear sclerosis cataract. Examination of the fundus revealed normal findings in both eyes. Because there were no signs of inflammation, the diagnosis of endothelial rejection was questioned, whereas the presence of significant corneal ectasia suggested the diagnosis of corneal hydrops. The significant corneal edema, however, prevented a proper assessment of the DM. This is where OCT of the anterior segment of the eye was used to evaluate the cornea, revealing the detachment of the DM underlying the corneal edema and confirming the diagnosis of acute hydrops (Figures 1 and 2).

The patient's right eye was treated conservatively with cycloplegic drops. The patient refused surgical interventions such as intracameral injection of air or sulfur hexafluoride (SF6) and elected to wait. At her next follow-up visit after 6 weeks, her eye showed marked improvement, with a visual acuity of $20 / 60$, paracentral scarring, no corneal edema, with complete resolution of the DM detachment visible on OCT (Figures 3 and 4).

${ }^{\star}$ Correspondence to: AL-Damri AL-Jawhara, King Saud Medical City, Riyadh, Saudi Arabia, E-mail: dr.aljawhara@gmail.com

Received: April 01, 2019; Accepted: April 19, 2019; Published: April 26, 2019 


\section{Discussion}

Ezra, et al. [4] reported two cases of corneal hydrops many years after PKP (25 years in one case, 30 years in the other) in 2007, where there was DM detachment with no signs of rejection in both cases. The edema cleared with residual scarring in the first patient, whereas edema did not clear in the second patient, who underwent another PKP. In cases of corneal hydrops after PKP, the authors suggest that edema is usually localized at the host-donor junction and involves both the donor cornea and the host cornea, whereas in graft failure or endothelial rejection the edema mainly involves the donor cornea [4].

Wickremasinghe, et al. [5] looked at the histology of the donor button in patients who had hydrops after PKP, which revealed breaks in the DM, scrolling of the free edges, and stromal edema. These findings are similar to those in patients with hydrops in non transplanted corneas [5].

Oshida, et al. [6] reported an interesting finding of acute hydrops that developed 7 years after PKP for keratoconus. The acute hydrops was localized to the host cornea without causing edema in the graft.

In the case described above, the corneal edema was localized; however, it involved the host cornea and the donor cornea, as shown in anterior segment OCT. We observed the patient for 6 weeks, after that her vision had improved to 20/60. The corneal edema cleared up with faint residual scarring that was not in the visual axis, and anterior segment OCT showed a normally attached DM.

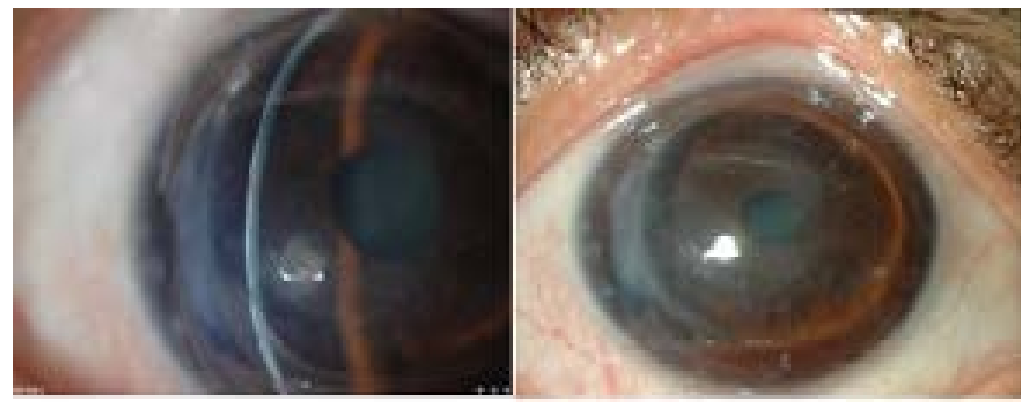

Figure 1. Slit lamp photographs of the anterior segment of the right eye show the localized inferotemporal corneal edema
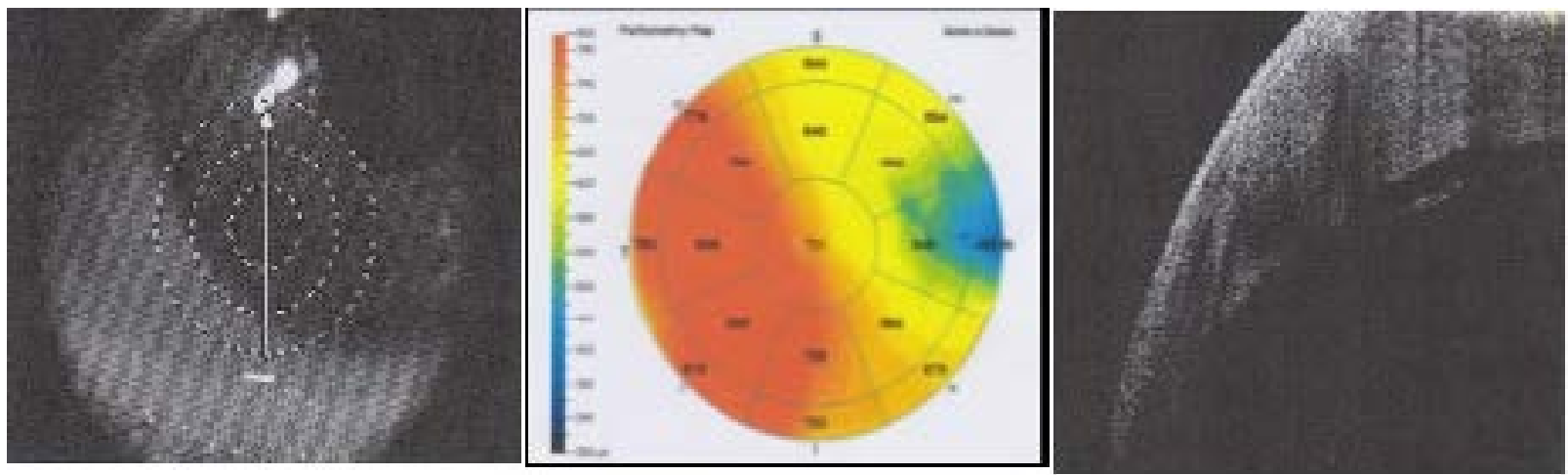

Figure 2. Optical coherence tomography images of the anterior segment of the right eye show the localized detachment of the descemet membrane
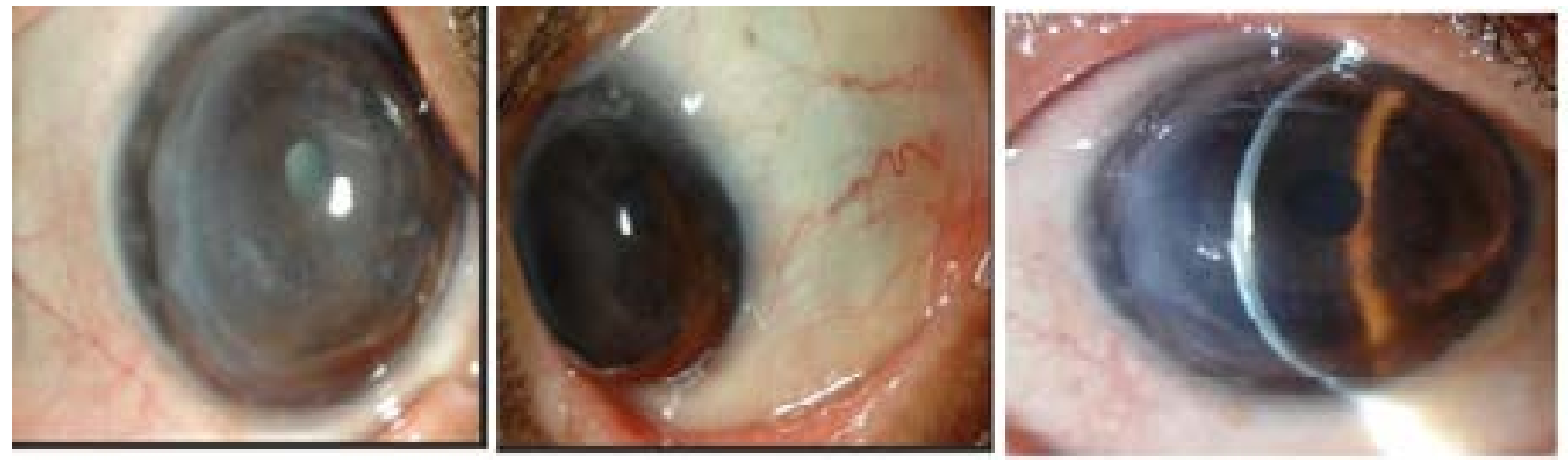

Figure 3. Slit lamp photographs of the anterior segment of the right eye show resolution of the corneal edema 
AL-Jawhara A (2019) The role of anterior segment optical coherence tomography (OCT) in the diagnosis of corneal hydrops after penetrating keratoplasty in a patient with keratoconus

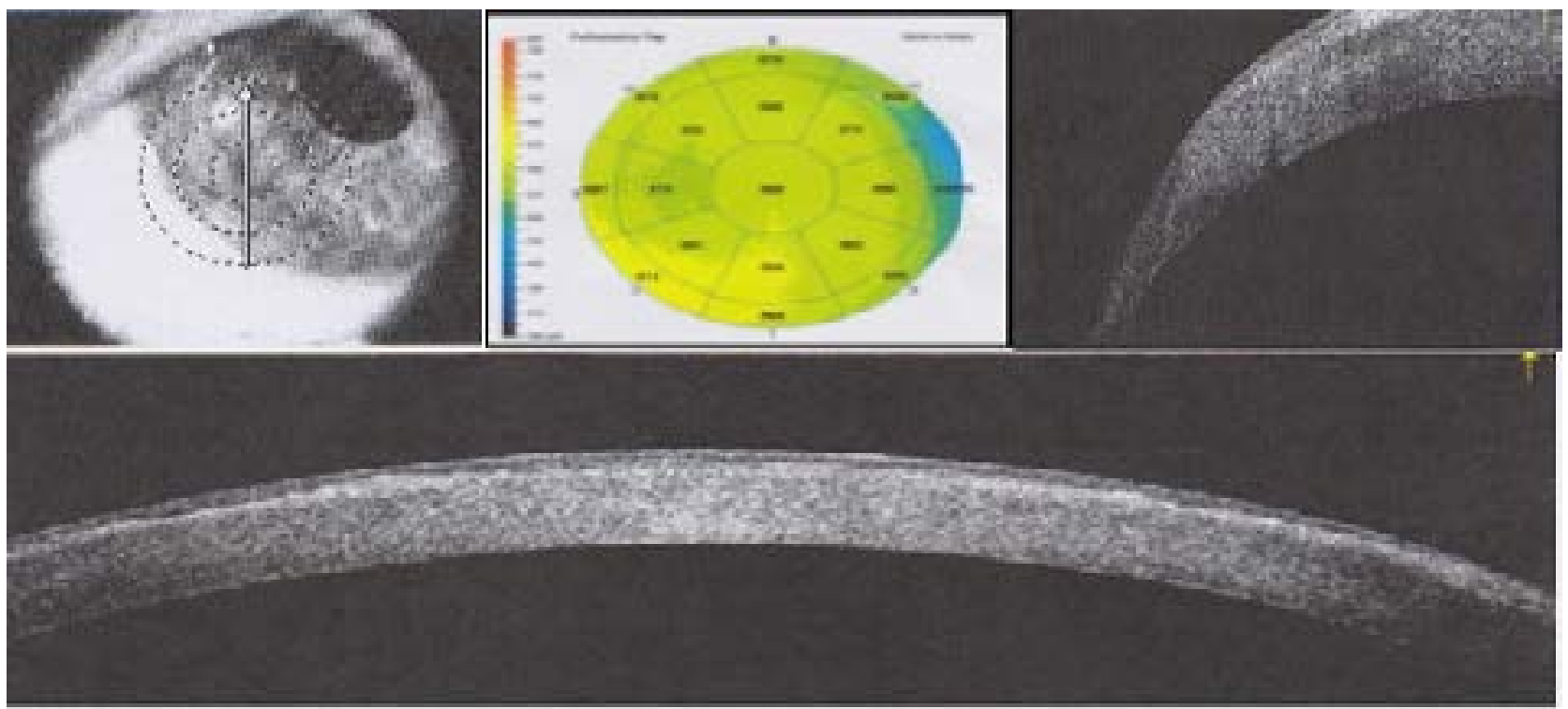

Figure 4. Optical coherence tomography images of the anterior segment of the right eye show that the descemet membrane is fully attached

\section{Conclusions}

Corneal hydrops post penetrating keratoplasty is a rare condition, especially in cases of significant ectasia with no signs of inflammation or rejection. This diagnosis eliminates the use of unnecessary intensive steroid therapy. Anterior segment OCT provides a way to confirm the diagnosis of DM detachment, which may be missed clinically in cases of significant corneal edema.

\section{References}

1. Tuft SJ, Gregory WM, Buckley RJ (1994) Acute corneal hydrops in keratoconus. Ophthalmology 101: 1738-1744. [Crossref]
2. Basu S, Vaddavalli PK, Ramappa M, Shah S, Murthy SI, et al. (2011) Intracameral perfluoropropane gas in the treatment of acute corneal hydrops. Ophthalmology 118: 934-399. [Crossref]

3. Grewal S, Laibson P, Cohen E, Rapuano C (1999) Acute hydrops in the corneal ectasias: associated factors and outcomes. Trans Am Ophthalmol Soc 97: 187-198. [Crossref]

4. Ezra DG, Mehta JS, Allan BD (2007) Late corneal hydrops after penetrating keratoplasty for keratoconus. Cornea 26: 639-640. [Crossref]

5. Wickremasinghe SS, Smith GT, Pullum KW, Buckley RJ (2006) Acute hydrops in keratoconus masquerading as acute corneal transplant rejection. Cornea 25: 739-741. [Crossref]

6. Oshida T, Fushimi N, Sakimoto T, Sawa M (2011) Acute hydrops in a host cornea after penetrating keratoplasty for keratoconus. Jpn J Ophthalmol 55: 418-419. [Crossref]

Copyright: @2019 AL-Jawhara A. This is an open-access article distributed under the terms of the Creative Commons Attribution License, which permits unrestricted use, distribution, and reproduction in any medium, provided the original author and source are credited. 